\title{
Social stress promotes changes in local metabolism of glucocorticoids
}

\author{
J. Pácha, P. Klusoňová, P. Ergang, M. Vodička \\ Institute of Physiology, Czech Academy of Science, Prague, Czech Republic
}

\section{Introduction and objectives}

Stress, a common aspect of modern life, produces profound physiological and behavioral disturbances that may contribute to many psychiatric disorders. The principal endocrine component of the stress response is the activation of the hypothalamic-pituitaryadrenocortical (HPA) axis, a self-regulatory pathway that utilizes its end products cortisol and corticosterone to control its own activation. Local effect of glucocorticoids does not depend only on its concentration but also on the enzyme $11 \beta$-hydroxysteroid dehydrogenase type 1 (11HSD1), which amplifies intracellular glucocorticoid concentration by conversion of the inactive cortisone and 11-dehydrocorticosterone to active hormones. As cytokines are potent modulators of 11HSD1, the aim of this study was to determine whether stress modulates 11HSD1 in lymphoid organs and whether these changes are correlated with local changes of cytokines.

\section{Methods}

Fisher 344 rats were exposed to chronic emotional homotypic stress (resident-intruder paradigm, 7 days) or short-term variable stress combining emotional and physical stressors (3 days). Plasma level of corticosterone, tumor necrotic factor $\alpha$ (TNF $\alpha$ ), and interleukin $1 \beta$ (IL-1 $\beta$ ) were measured by commercial kits, expression of $11 \mathrm{HSD} 1$, TNF $\alpha$ and IL-1 $\beta$ mRNAs by quantitative RT-PCR and 11-reductase activity of $11 \mathrm{HSD} 1$ by radiometric assay. MLN, mesenteric lymphatic nodes

Fig. 1

\section{Results}
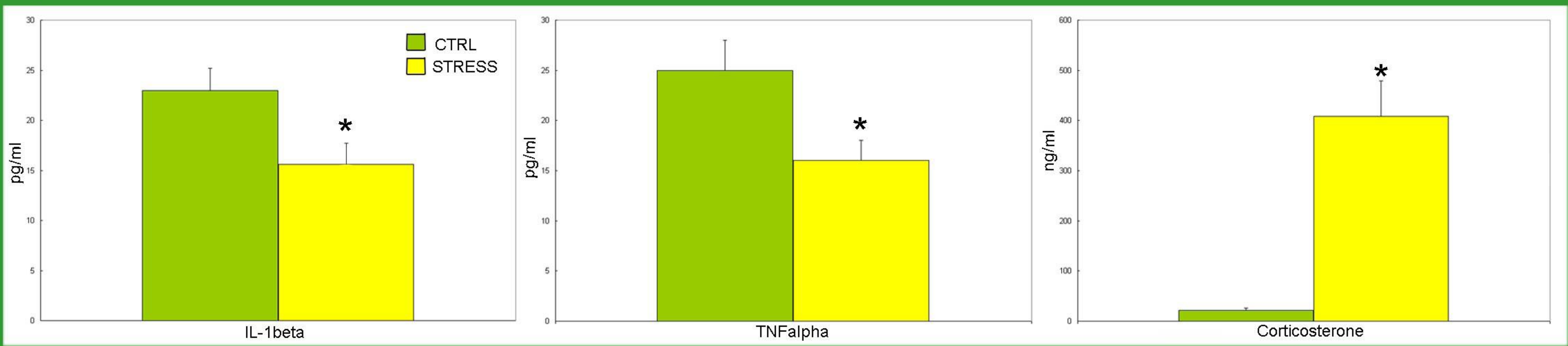

Effect of emotional stress on plasma level of TNF $\alpha$, IL-1 $\beta$, and corticosterone.

Fig. 2

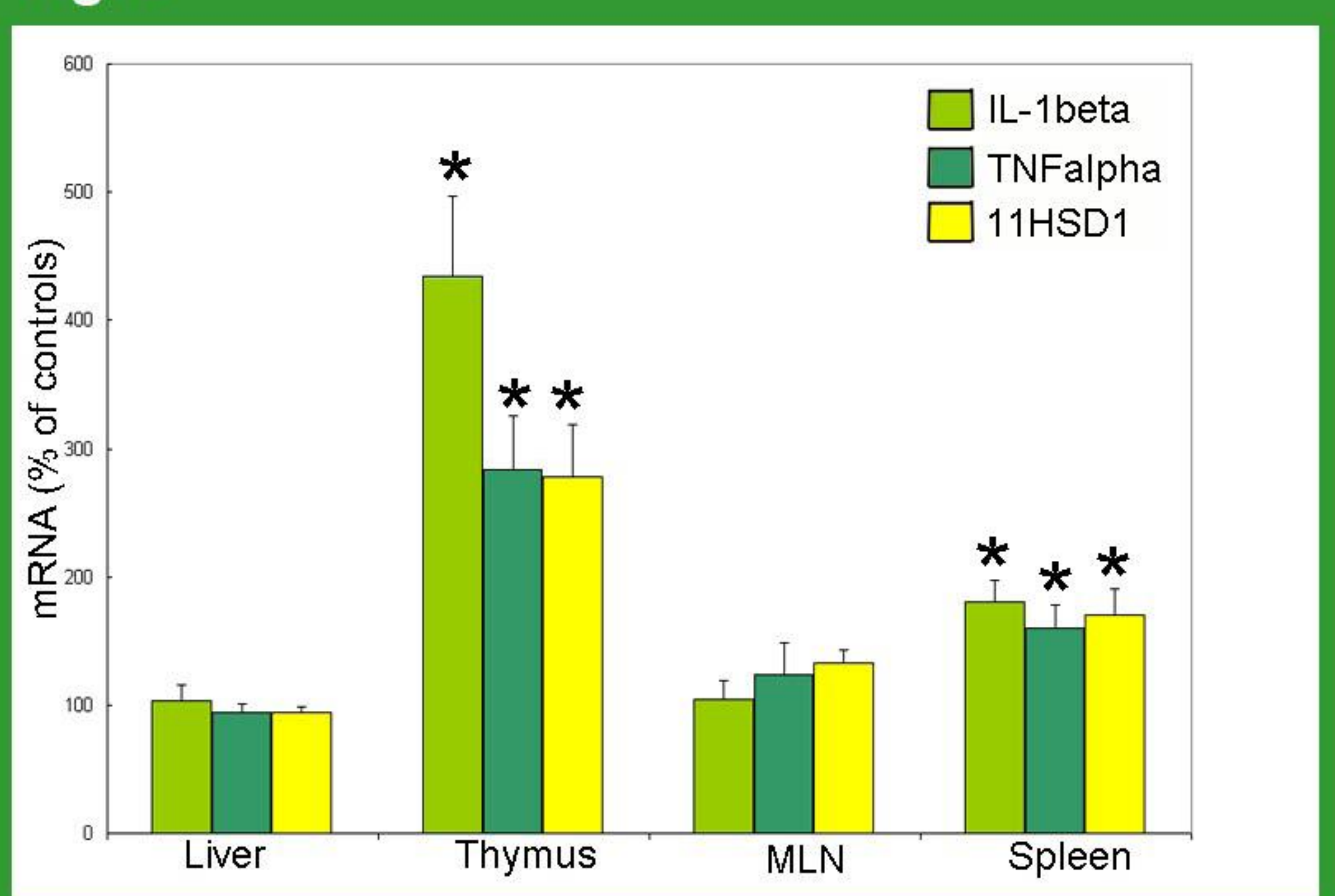

Effect of emotional stress on mRNA expression of TNF $\alpha$, IL-1 $\beta$ and 11HSD1.

\section{Fig 4.}

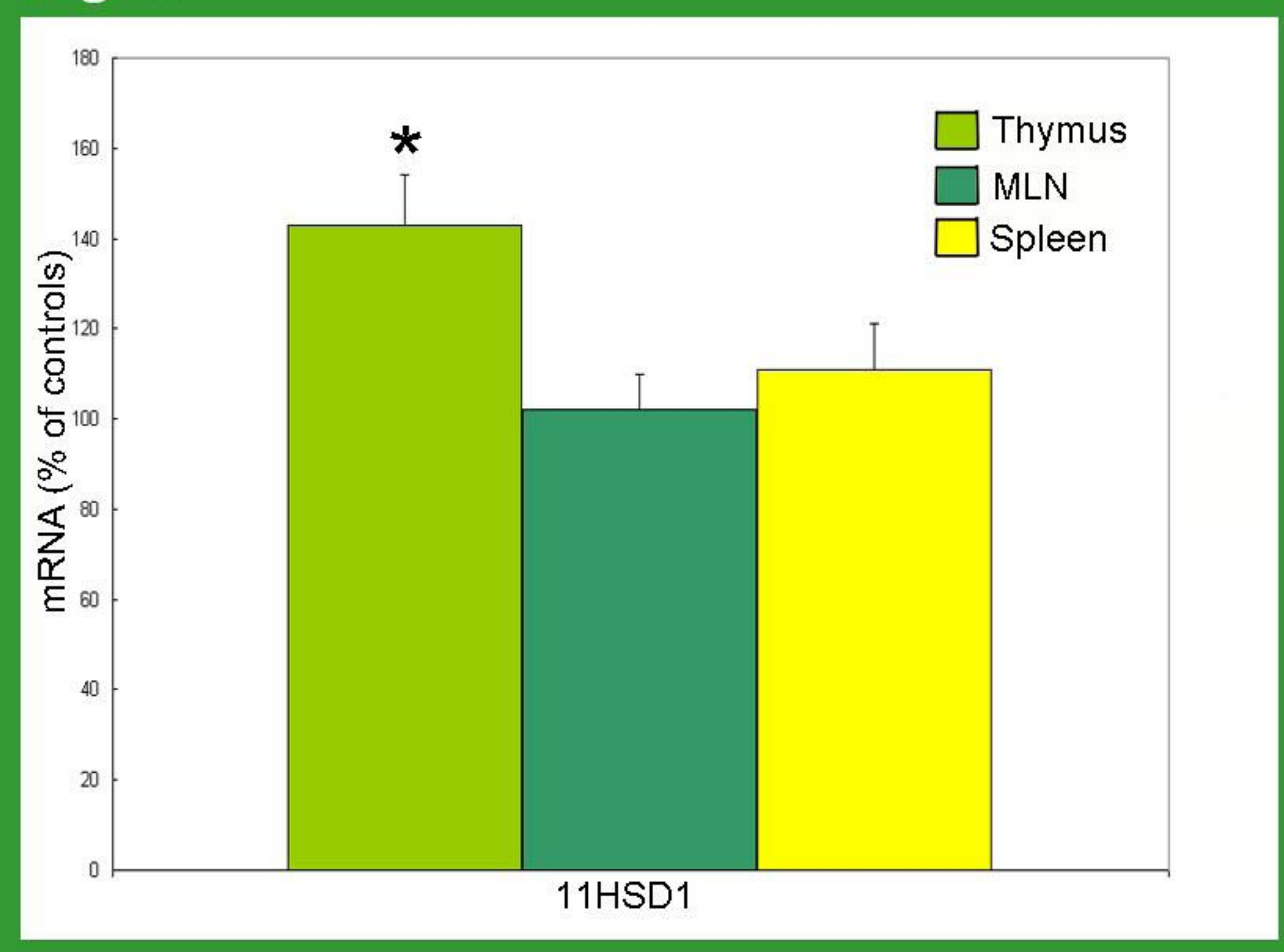

Effect of short-term variable stress on expression of 11HSD1 mRNA.
Fig. 3

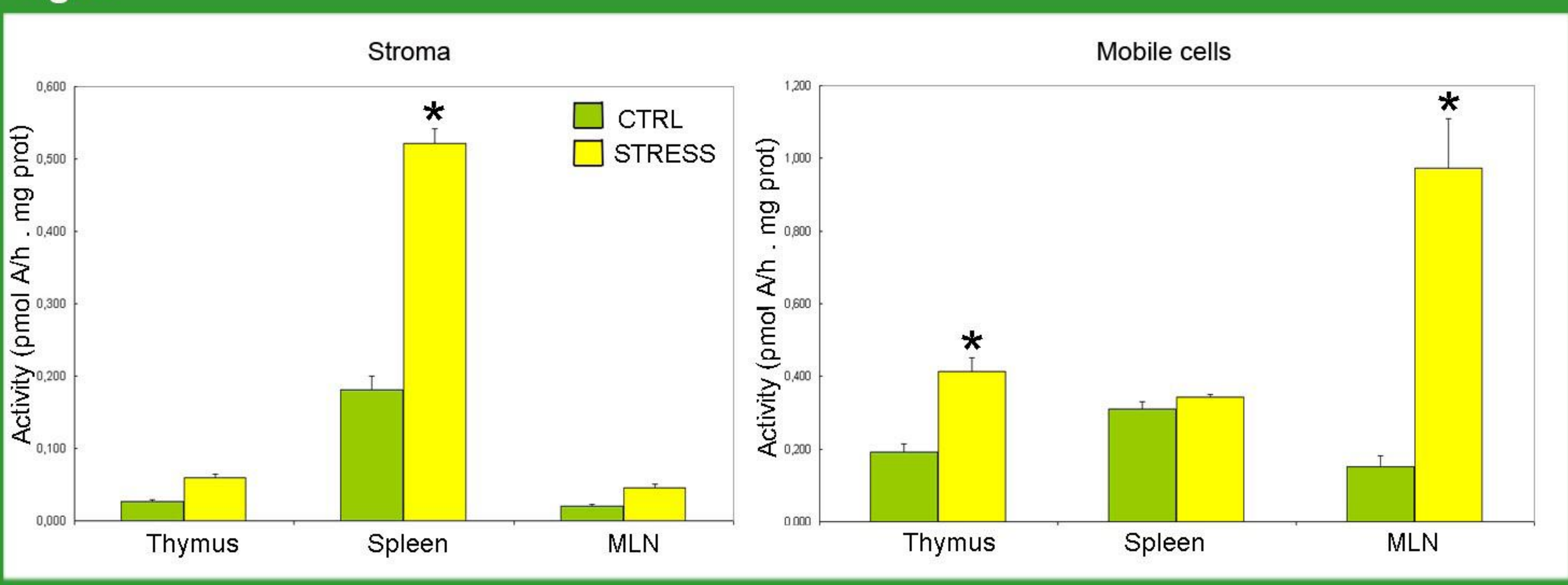

Effect of previous emotional stress on 11-reductase activity of lymphoid organs in mobile and stroma cells cultivated in vitro.

\section{Conlusions}

Powerful stressors modulate glucocorticoid signaling in primary and secondary lymphoid organs and this effect seems to be associated with local upregulation of TNF $\alpha$ and IL-1 $\beta$. 